\title{
Pancreatic Cysts: Diagnostic Role of EUS-Guided Microforceps Biopsy and Confocal Laser Endomicroscopy
}

\author{
Darina Kohoutova (D, ${ }^{1,2}$ Sameer Zar, ${ }^{1}$ Rudolf Repak, ${ }^{2}$ Panagiotis Vlavianos, ${ }^{3}$ \\ and Jan Bures $\mathbb{D}^{2}$ \\ ${ }^{1}$ The Royal Marsden Hospital NHS Foundation Trust, Fulham Road, Chelsea, SW3 6JJ London, UK \\ ${ }^{2}$ 2nd Department of Internal Medicine-Gastroenterology, Charles University, Faculty of Medicine in Hradec Kralove, \\ University Hospital, Sokolska 581, Hradec Kralove 500 05, Czech Republic \\ ${ }^{3}$ Hammersmith Hospital, Imperial College Healthcare NHS Trust, London, UK
}

Correspondence should be addressed to Darina Kohoutova; darina.kohoutova@seznam.cz

Received 15 May 2019; Revised 22 July 2019; Accepted 6 August 2019; Published 12 September 2019

Academic Editor: Konstantinos Triantafyllou

Copyright (c) 2019 Darina Kohoutova et al. This is an open access article distributed under the Creative Commons Attribution License, which permits unrestricted use, distribution, and reproduction in any medium, provided the original work is properly cited.

\begin{abstract}
Frequent use of high-quality cross-sectional imaging has led to a significant rise in diagnosis of pancreatic cystic lesions (PCLs). Despite the fact that enormous effort has been put into the research of PCLs within the last two decades and multiple guidelines have been developed, our clinical decision-making especially in regard to mucinous lesions remains limited. Currently, clinical assessment, cross-sectional imaging and EUS with fluid analysis (if appropriate) belong to the standard care in patients with PCLs. For differentiation of mucinous from nonmucinous cysts, the sensitivity of cytological investigation and CEA in the cyst fluid is $42 \%$ and $52-79 \%$, respectively. Due to the limited accuracy, further diagnostic tools are warranted. Two EUS-guided approaches have been introduced recently. Through-the-(19-gauge EUS) needle Moray microforceps have been developed, and several studies have acknowledged their contribution to the correct diagnosis as they help to overcome limited cellularity of the EUS-guided cyst fluid aspiration and traditional cytology. Confocal laser endomicroscopy offers real-time images and seems to be a promising method for the diagnosis and differential diagnosis of pancreatic PCLs. Example images of the needle-based confocal laser endomicroscopy criteria for the diagnosis of PCLs have been suggested recently. Before both, Moray microforceps and confocal laser endomicroscopy can be widely accepted, further studies are necessary to determine the real diagnostic yield and the clinical efficacy.
\end{abstract}

\section{Introduction}

Frequent use of high-quality cross-sectional imaging has led to a significant rise in diagnosis of pancreatic cystic lesions (PCLs). The recent meta-analysis has confirmed pooled prevalence of $8 \%$ in asymptomatic individuals [1]. Incidence of PCLs increases with age and reaches $37 \%$ in patients aged $>80$ years [2]. It has been acknowledged that individuals with PCLs have a significantly higher overall risk of pancreatic cancer [3]; nevertheless, clinicians face a challenge how to optimize management of individuals with a PCL, when currently insufficient diagnostic tools are taken into account [4]. Patients should not be overtreated with surgery and on the contrary, individuals with a malignant PCL should not be kept under surveillance inappropriately [5].

The aim of our paper is to review classification of pancreatic cysts and to discuss the role of the most recent EUS- (endoscopic ultrasound-) guided diagnostic options for PCLs.

\section{Classification of PCLs and Current Knowledge}

Pancreatic cystic lesions are divided into mucinous lesions, including mucinous cystic neoplasm (MCN) and intraductal papillary mucinous neoplasm (IPMN) and nonmucinous 
TABLE 1: IPMN: intraductal papillary mucinous neoplasm; MCN: mucinous cystic neoplasm; SCN: serous cystic neoplasm; SPN: solid pseudopapillary neoplasm; p: predominantly; HOP: head of pancreas.

\begin{tabular}{lccccc}
\hline & IPMN & MCN & SCN & Pancreatic pseudocyst & SPN \\
\hline Male & $60-70 \%$ & $5 \%$ & $10 \%$ & $75 \%$ & $10 \%$ \\
Age (years) & $60-70$ & $40-50$ & 70 & Around 50 & Any localisation \\
Localization & p HOP & p body and tail & p tail & Any localisation \\
Communication with PD & Yes & No & No & No \\
Cytology & Mucinous cells & Mucinous cells & & Inflammatory cells \\
CEA in cyst & High & High & Low & Low & No \\
Mucin in cyst & Yes & Yes & No & High & No \\
Amylase in cyst & High & Low & Low & Low \\
\hline
\end{tabular}

lesions which include serous cystic neoplasm (SCN), pseudocyst, cystic neuroendocrine tumour, solid pseudopapillary tumour, and cystic pancreatic ductal adenocarcinoma [6-8]. Basic characteristics of PCLs are summarized in Table 1. Mucinous cystic lesions belong, together with pancreatic intraepithelial neoplasia, to the precursor lesions for pancreatic adenocarcinoma [9].

Patients with symptomatic PCLs can present with jaundice, recent onset of type 3 diabetes, (recurrent) pancreatitis, anorexia, weight loss, abdominal/back pain, nausea, and/or vomiting $[7,8]$. Clinical assessment, cross-sectional imaging and EUS with fluid analysis, if appropriate (cytology, CEA), play the major role in current standard care in patients with a PCL. MRI/MRCP has been proven to be superior to CT in identifying communication between a PCL and the pancreatic ductal system and the presence of a mural nodule and in identifying if a patient has single or multiple PCLs [10-13]. In a recent meta-analysis, cytological investigation of the cyst fluid had $42 \%$ sensitivity and $99 \%$ specificity for differentiation of mucinous from the nonmucinous PCLs [14]. Cyst fluid CEA $\geq 192$ $\mathrm{ng} / \mathrm{mL}$ can differentiate mucinous from nonmucinous cyst with a sensitivity of $52-78 \%$ and specificity of $63-91 \%$. Cytology and/or cyst fluid CEA level is not helpful in differentiation between MCN and IPMN [10, 15]. Despite the fact that the evidence is weak, antibiotic prophylaxis prior to an EUS-guided FNA of PCLs and 3-5 days after keeps being used routinely. Guarner-Argente et al. [16] and recently Facciorusso et al. [17] have not observed reduction of risk of infection after antibiotic prophylaxis. In view of this, further prospective studies are warranted with the aim to abandon routine periprocedural use of antibiotics.

\subsection{Intraductal Papillary Mucinous Neoplasia (IPMN).} IPMNs are classified into the main duct (Figures 1 and 2 ), mixed type, and branch duct neoplasias according to the communication with and involvement of the main and/or branch pancreatic ductal system [9]. Majority of IPMNs are solitary and are localized in the pancreatic head, yet $20-40 \%$ are multifocal [18]. Typically, males (in $60-70 \%)$ of age $60-70$ years would be diagnosed with an IPMN. The absence of capsule, communication with the pancreatic duct, presence of mucin, and cystic fluid high in CEA and amylase belong to the main features of

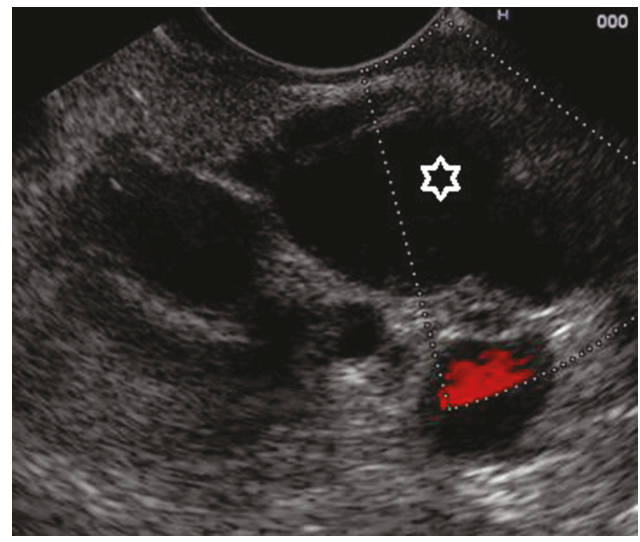

FIgURE 1: Main duct intraductal papillary mucinous neoplasia (asterisk: dilatation of the main pancreatic duct).

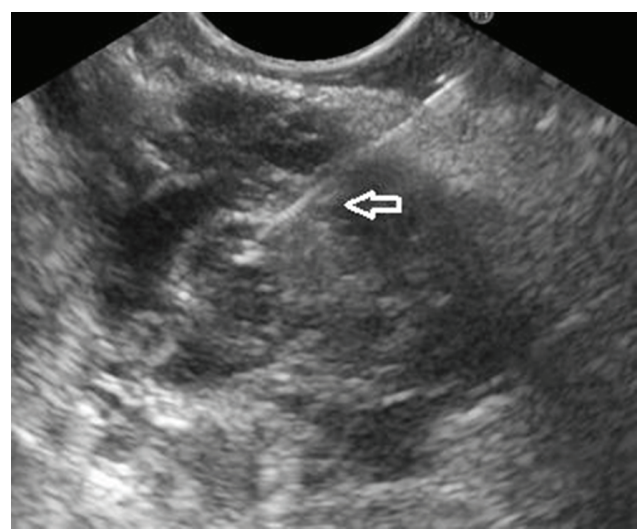

FIgURE 2: Main duct intraductal papillary mucinous neoplasia, FNA performed (arrow pointing at the FNA needle).

IPMNs [19]. Based on histological characteristics and immunohistochemical reactivity for mucins (MUC), IPMNs are classified into gastric (less aggressive phenotype, usually originating in branch ducts), intestinal, pancreatobiliary, and oncocytic (more aggressive phenotypes, usually originating in the main pancreatic duct) types [9, $18,20,21]$. Invasive carcinomas related to IPMN can be either colloid or tubular (conventional), and there is a clear evidence that the colloid carcinomas have a better 
prognosis than the tubular carcinomas [22-24]. The most typical mutations observed in IPMNs are those in oncogenes KRAS and GNAS and in tumour suppressor gene RNF43 [6]. Due to the risk of malignant transformation, patients with main duct IPMNs and mixed type IPMNs should be considered for surgery. According to the recent European guidelines, the absolute indications for surgery are tumour-related jaundice, solid mass, presence of an enhancing mural nodule $(\geq 5 \mathrm{~mm})$, positive cytology for malignant/high grade dysplasia, and/or main pancreatic duct dilatation $\geq 10 \mathrm{~mm}$ [10].

2.2. Mucinous Cystic Neoplasm (MCN). MCNs are usually solitary large unilocular cysts predominantly found in the body or the tail of the pancreas in 40-50-year-old females (Figure 3). Cysts are characterized by the absence of communication with the pancreatic ductal system [19]. Peripheral "eggshell" calcification is seen in less than $20 \%$ of MCNs; nevertheless, such a finding is specific for a mucinous cystic neoplasm and is highly predictive of malignancy $[25,26]$. Further typical features are the presence of ovarian-like stroma (with expression of hormone receptors) and mucinproducing epithelium. Cystic fluid is high in CEA and low in amylase $[6,25]$. Mutation in KRAS oncogene is the most commonly found mutation; GNAS mutation is not observed in $\mathrm{MCN}-$ on contrary to an IPMN [27]. As MCN is usually discovered in younger patients in the body or the tail of the pancreas and has clearly a malignant potential, majority of centres will recommend surgery [25]. The recent European guidelines suggest resection for all patients with a $\mathrm{MCN} \geq$ $40 \mathrm{~mm}$ in size or who are symptomatic or have risk factors (such as a mural nodule), irrespective of the size [10].

2.3. Serous Cystic Neoplasm (SCN). SCN are benign cystic lesions predominantly found in the tail of the pancreas in females aged around 70 years (Figures 4 and 5). Imaging usually shows microcystic "honeycomb-like" lesion with central scar and central calcification with no communication with the pancreatic duct. The absence of mucin in the cyst, low CEA, and low amylase in the cyst fluid are characteristics of SCN $[19,25]$. Mutations in VHL gene are typical for serous cystic lesions [28]. Surgical treatment should be proposed only if the diagnosis remains uncertain after a complete workup, if significant and related symptoms are present (jaundice, pancreatitis, and gastric outlet obstruction), or if exceptionally, a concern regarding malignancy arises [25, 29].

2.4. Pseudocyst. Pancreatic pseudocysts can be present anywhere in the pancreas and are more frequently found in males, usually in association with chronic pancreatitis (Figures 6 and 7). CEA is low, no mucin, and no molecular markers related to malignancy can be detected $[6,25]$. Communication with the pancreatic duct is usual; therefore, the content is rich in amylase. Amylase $<250 \mathrm{U} / \mathrm{L}(4.2 \mu \mathrm{kat} / \mathrm{L})$ may exclude the presence of a pseudocyst with a sensitivity $44 \%$ and specificity $98 \%$ [30].

2.5. Solid Pseudopapillary Tumour (SPT). SPTs, rare lesions, are typically identified in young females and express proges-

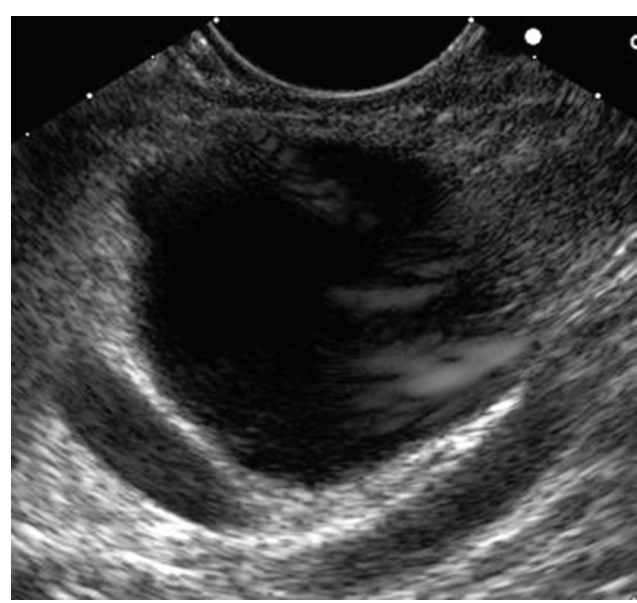

FIgUre 3: Mucinous cystic neoplasm. CEA: $242 \mathrm{ng} / \mathrm{mL}$. Cytology benign.

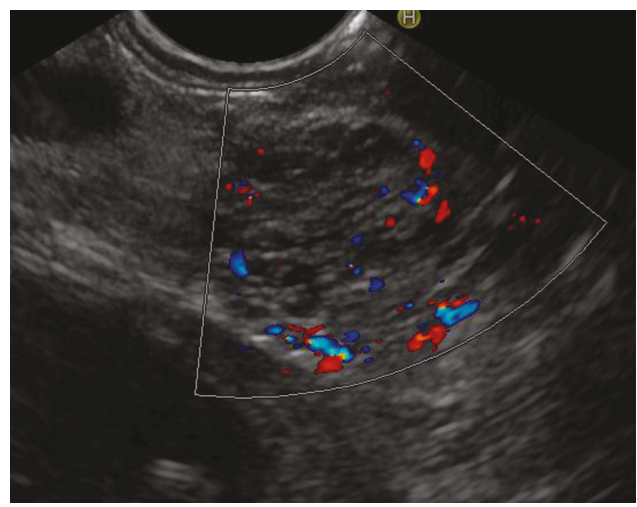

FIgURE 4: Serous cystic neoplasm with vascular septa.

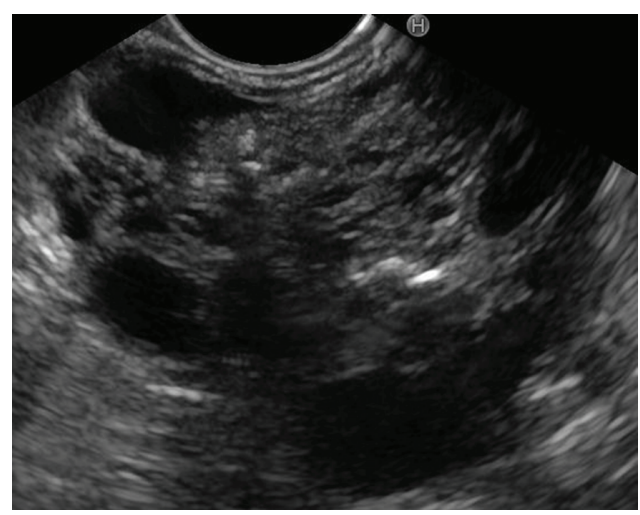

Figure 5: Serous cystic neoplasm with micro- and macrocysts.

terone and estrogen receptors (Figure 8). They can be found anywhere in the pancreas and usually consist of mixed solidcystic lesions. There is no communication with the pancreatic duct; the cyst fluid is low in CEA and amylase [6, 19, 25]. SPTs belong to slowly growing tumours with low malignant potential and infrequent metastases [31]. Surgical resection is warranted [25].

Enormous effort has been put into the research regarding pancreatic cystic lesions within the last two decades; nevertheless, diagnostic accuracy, as shown above, is rather 


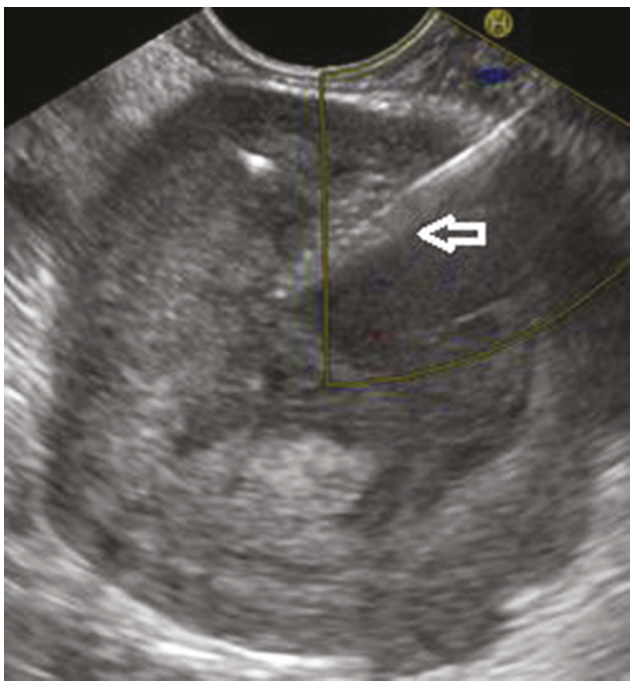

Figure 6: Pancreatic pseudocyst (FNA had been performed before the AXIOS stent was inserted; arrow pointing at the FNA needle).

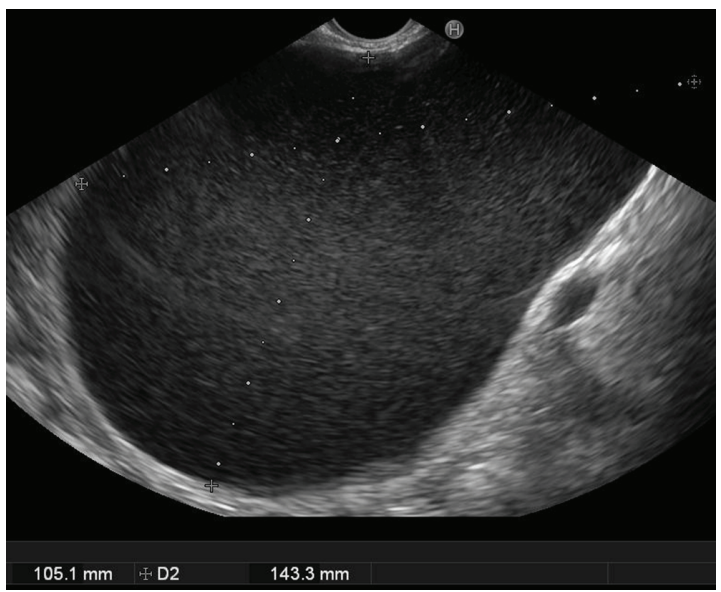

FIGURE 7: Large pancreatic pseudocyst $(143 \times 105 \mathrm{~mm})$.

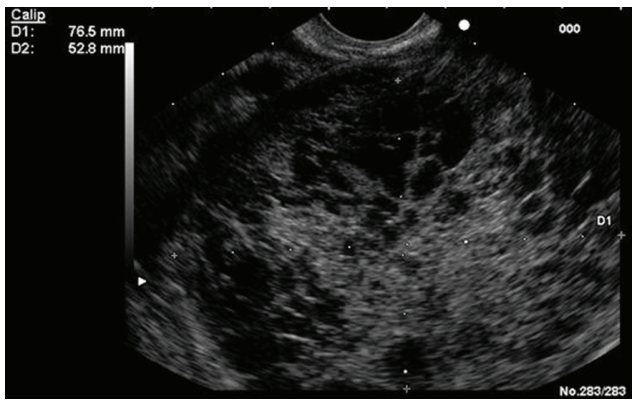

FIgURE 8: Solid-cystic pseudopapillary tumour.

poor. Multiple guidelines have been developed (including Sendai [32], Fukuoka [22], revised Fukuoka [33], American Gastroenterological Association (AGA) [34], and European guidelines [10]); still, our current clinical decision-making especially in regard to mucinous lesions remains limited. Further diagnostic tools are warranted. We offer an update on two EUS-guided methods, and we discuss their role in the PCL diagnosis.

\section{EUS-Guided Microforceps Biopsy}

In an attempt to improve the diagnostic yield of PCLs, through-the-needle direct intracystic biopsy and pancreatic cystoscopy were first performed in 2010 [35]. Biopsy forceps and a SpyGlass fiber optic probe were passed through the 19-gauge EUS needle in two patients with a PCL in the head of the pancreas. Diagnosis of a mucinous lesion was established in both cases. One patient developed severe acute pancreatitis one month after the biopsies, which was rather not associated with the procedure [35]. Another report came in 2015, when Barresi et al. documented a contribution of miniforceps biopsy to the diagnosis of a mucinous tumour in the body of the pancreas in a 46-year-old woman. Biopsies were taken from the wall of the cyst [36]. Subsequently, novel through-theneedle Moray microforceps have been developed, and Pham et al. reported a first successful biopsy of an intracystic nodule leading to a diagnosis of a mucinous cyst [37]. Further, authors have confirmed in individual cases that Moray microforceps can be useful in determination of the nature of the PCLs and can contribute to their management and risk stratification [38-41]. Moray microforceps are $230 \mathrm{~cm}$ in length with a jaw opening width of $4.3 \mathrm{~mm}$ and a sheath of $0.8 \mathrm{~mm}$ in diameter that easily passes through a 19-gauge EUS-FNA needle [38]. The role of Moray microforceps in the preoperative diagnosis has been acknowledged subsequently [42-44].

A first larger study, retrospective in design, which involved 27 patients with PCLs, was published in 2018: 14 patients with cysts located in the pancreatic head and/or uncinate process and 13 patients with cysts located in the body and/or tail of the pancreas were enrolled. Moray microforceps were passed through the 19-gauge needle under the EUS guidance, and 3-4 subsequent samples were taken from the cyst wall and placed into formalin. After completion of biopsies, cyst fluid was aspirated and sent for cytology and CEA level analysis. Microforceps biopsies were technically successful in all 27 cases and provided a pathology diagnosis in 24 of 27 cases. No periprocedural adverse event was recorded (including bleeding, infection, perforation, and pancreatitis). Overall, microforceps biopsy results changed the diagnosis in 7 patients; nevertheless, cytology provided a diagnosis of a mucinous cyst in $4 / 27$, and these have not been detected by microforceps biopsies. The authors therefore concluded that Moray forceps could be a useful adjunctive tool, which would be complementary to existing EUS-FNA sampling protocols for PCLs [45]. Also in 2018, Basar et al. published data on 42 patients from a multicentre study: they confirmed that Moray microforceps biopsy was far superior to cytology in providing a specific cyst diagnosis [46]. A similar conclusion came from Zhang et al.: pancreatic cyst fluid analysis and microforceps biopsy have comparable results in distinguishing between mucinous and nonmucinous cysts and for detecting high-risk cysts; 
nevertheless, similar to the study performed by Basar et al. [46], microforceps biopsy has been superior for diagnosing specific cyst subtypes [47]. Another most recent study published on microforceps biopsy by Kovacevich et al. has shown promising results, nevertheless, three adverse events (11\%) have been recorded [48].

In conclusion, dedicated through-the-needle Moray microforceps allow biopsy of the PCL wall or a mural nodule. This helps to overcome the limited cellularity of the EUSguided cyst fluid aspiration and traditional cytology. Further, it can provide guidance when at best modest accuracy of CEA is taken into account. Yet, the precise role of microforceps biopsy remains to be defined by large prospective studies before routine clinical implementation is recommended.

\section{Confocal Microscopy}

The principle of confocal laser scanning microscopy is not new; it was invented as early as 1957 [49]. Subsequent use in gastroenterology started in the mid-1990s [50]. The first generation of dedicated endoscopes enabled the introduction of confocal laser endomicroscopy (CLE) in the late nineties. A confocal endomicroscope was miniaturised to a size that made it possible to be integrated in the distal end of a high-resolution videoendoscope. A lot of research work was done afterwards, both experimental and clinical [51]. CLE classification was suggested for Barrett's oesophagus and colorectal neoplasia [51,52]. Our group studied experimental pharmacokinetics, and organ distribution of fluorescein determined the optimum time interval for diagnostic scanning (5-10 minutes after the fluorescein administration) and found high concentration in all organs of the gastrointestinal tract (including the pancreas), necessary for optimal confocal laser imaging [53]. Recently, single miniaturised CLE probes have become commercially available. These probes can be introduced through a working channel of a conventional videoendoscope into the lumen of the gastrointestinal tract or through a 19-gauge needle for fine-needle-based CLE. They enable observation of the inner wall of pancreatic cystic lesions during an endoscopic ultrasound-guided fine-needle aspiration [6, 51, 54-58].

Since 2010 , more than fifty papers have been published, including ESGE $[54,59]$ and ASGE technology reviews [51] and a meta-analysis [60]; however, only few clinical studies have been accomplished so far [61-68]. Konda et al. [61] published their first experience with a prototype confocal laser probe. Eighteen patients (with 16 cysts and 2 mass lesions) were investigated in this multicentre feasibility study at a tertiary setting. CLE was technically feasible (in 17 of 18 cases) using a 19-gauge needle under EUS guidance. There were no device malfunctions; two cases were complicated with acute pancreatitis. The diagnosis was confirmed with histology or positive cytology in 10 out of 18 patients [61]. The INSPECT study investigated 66 patients (images were available for 65) in USA, Germany, and France. The authors aimed to define criteria for differentiation of mucinous and nonmucinous cystic lesions. An epithelial villous structure on confocal images was associated with mucinous cysts significantly [62]. Eighteen patients with indeterminate pan- creatic duct strictures were investigated prior to surgery in another study [63]. Real-time CLE images were obtained during ERCP. Cytology or histopathology in 15 of 16 cases showed similar results to CLE interpretation. Agreement between cytology or histopathology and CLE was high $(\kappa=0.8)$. Pancreatic CLE changed management in four patients [63]. The DETECT study combined EUS-guided through-the-needle direct visualisation (SpyGlass fiber optic probe) and probe-based CLE inserted through a 19-gauge needle. Thirty patients with pancreatic cystic neoplasms were enrolled. The combination of cystoscopy and CLE of pancreatic cysts might have strong concordance with the clinical diagnosis of pancreatic cystic neoplasms (sensitivity 87, specificity 77 , and positive and negative predictive values 100\%) [64]. Napoleon et al. [65] investigated 31 patients with pancreatic cysts and identified criteria for the diagnosis of serous cystadenoma [65]. Twenty patients with pancreatic cystic neoplasms were investigated within a 16 -month period in another study [66]. The procedure and confocal image acquisition were successful in $90 \%$. The sensitivity, specificity, and diagnostic accuracy were 66,100 , and $80 \%$. No complications were recorded [66]. The pancreatic cyst epithelial wall can be visualised successfully by CLE also in ex vivo surgical specimens [67]. Krishna et al. [68] investigated ten patients for the reproducibility of the in vivo endoscopic ultrasound-guided needle-based CLE image patterns in an ex vivo setting. Both in vivo (preoperative) and ex vivo confocal laser imaging of the surgically resected pancreatic cystic lesions correlated with surgical histopathology [68].

Example images of the needle-based CLE criteria for the diagnosis of PCLs have been suggested: (a) serous cystadenomas with the "superficial vascular network" criterion; (b) intraductal papillary mucinous neoplasms with the "papillae" criterion; (c) mucinous cystadenomas with the "epithelial border" criterion; (d) neuroendocrine neoplasms with the "dark aggregates of cells surrounded by gray areas of fibrosis and vessels" criterion; and (e) pseudocysts with the "field of bright, gray, or black particles" criterion [56, 65, 66] (Figure 9). For the near future, it will be mandatory to set a validated interpretation system of the CLE of pancreatic cystic lesions, to establish a unified training programme and to create a close standardized cooperation of gastroenterologists/endoscopists and pathologists. There is conflicting data on the reproducibility and accuracy of needle-based CLE in the available literature. In a multicentre US study, interobserver agreement of needle-based CLE recordings of 15 patients was low; the mean accuracy of observers was only $46 \%$. Interobserver agreement for the final diagnosis was poor $(\kappa=0.13)$ [69]. According to another project [70], interobserver agreement, intraobserver reliability, and diagnostic accuracy were high in differentiation of mucinous versus nonmucinous pancreatic cystic lesions. In a study with 29 consecutive patients (between 2013 and 2016), the overall sensitivity, specificity, and accuracy for the diagnosis of mucinous lesions were 95\%, 94\%, and 95\%, respectively. The interobserver agreement and intraobserver reliability were high ( $\kappa=0.81$ and $\kappa=0.86$, respectively). Similar results 


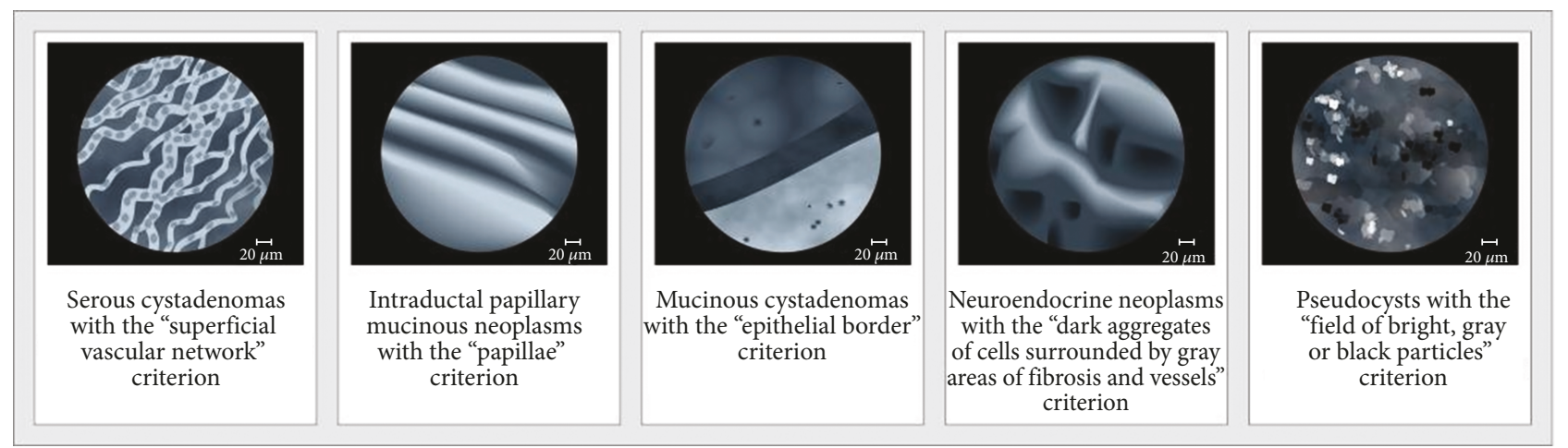

Figure 9: Confocal laser endomicroscopy for evaluation of pancreatic cystic lesions. Adopted from Napoleon et al. [65], Napoleon et al. [56], and Kadayifci et al. [66]. Drawing: Hana Kotlandova.

were achieved for recognizing the characteristic image pattern of serous cystadenomas $(\kappa=0.83$ and $\kappa=0.85)$. The overall specificity, sensitivity, and accuracy for the diagnosis of serous cystadenomas were $99 \%, 98 \%$, and $98 \%$, respectively [70].

Safety of needle-based CLE refers either to EUS-guided puncture of the cyst lesions or to side effects of i.v. fluorescein administration. Acute pancreatitis can complicate up to $4 \%$ of procedures; bleeding associated with a needle puncture is another possible complication [65]. A series of 2272 patients with CLE revealed no serious adverse complications of i.v. fluorescein administration. Mild adverse events (in 1.4\%) included nausea, vomiting, transient hypotension, diffuse rash, and mild epigastric pain [71].

Financial aspects must be also considered. Needlebased CLE of pancreatic cyst lesions increases the cost of the diagnostic process significantly [51]. On the other hand, needle-based CLE used with EUS-based fine-needle aspiration and/or biopsy might improve diagnostic accuracy, helping to reduce unnecessary surgery and patient follow-up, thus resulting in significant economic benefits by reducing the incidence of misdiagnosis owing to improved diagnostic accuracy [72]. Before the technology can be widely accepted, further studies are necessary to determine the real clinical efficacy and to evaluate the cost-effectiveness [51].

CLE seems to be a promising method for the diagnosis and differential diagnosis of pancreatic cystic lesions. Nevertheless, the common use is limited by its high cost and need of specific operator training (as a standardized institutional programme). That is why CLE is not easily available yet. Further studies are needed to evaluate the real diagnostic yield, cost-effectiveness, and health care economic analyses prior to implementation into a routine clinical practice.

\section{Conclusions}

Based on the current clinical practice, diagnostic accuracy for pancreatic cystic lesions is modest at best. Further diagnostic tools are warranted. Two EUS-guided methods have been introduced recently: Moray microforceps and CLE. They seem to be promising in regard to diagnostic yield; neverthe- less, further studies are warranted to determine clinical efficacy and to evaluate the cost-effectiveness.

\section{Conflicts of Interest}

The authors declare that they have no conflicts of interest.

\section{Acknowledgments}

This work was supported by the project PERSONMED-Centre for the Development of Personalized Medicine in Age-Related Diseases, Reg. Nr. CZ.02.1.01/0.0/0.0/17_048/0007441.

\section{References}

[1] G. Zerboni, M. Signoretti, S. Crippa, M. Falconi, P. G. Arcidiacono, and G. Capurso, "Systematic review and meta-analysis: prevalence of incidentally detected pancreatic cystic lesions in asymptomatic individuals," Pancreatology, vol. 19, no. 1, pp. 2-9, 2019.

[2] J. J. Farrell, "Prevalence, diagnosis and management of pancreatic cystic neoplasms: current status and future directions," Gut Liver, vol. 9, no. 5, pp. 571-589, 2015.

[3] S. Munigala, A. Gelrud, and B. Agarwal, "Risk of pancreatic cancer in patients with pancreatic cyst," Gastrointestinal Endoscopy, vol. 84, no. 1, pp. 81-86, 2016.

[4] H. Matthaei, G. Feldmann, P. Lingohr, and J. C. Kalff, "Molecular diagnostics of pancreatic cysts," Langenbeck's Archives of Surgery, vol. 398, no. 8, pp. 1021-1027, 2013.

[5] N. de Pretis, S. Mukewar, A. Aryal-Khanal, Y. Bi, N. Takahashi, and S. Chari, "Pancreatic cysts: diagnostic accuracy and risk of inappropriate resections," Pancreatology, vol. 17, no. 2, pp. 267-272, 2017.

[6] F. Li, A. Malli, Z. Cruz-Monserrate, D. L. Conwell, and S. G. Krishna, "Confocal endomicroscopy and cyst fluid molecular analysis: comprehensive evaluation of pancreatic cysts," World Journal Gastrointestinal Endoscopy, vol. 10, no. 1, pp. 1-9, 2018.

[7] T. Jana, J. Shroff, and M. S. Bhutani, "Pancreatic cystic neoplasms: review of current knowledge, diagnostic challenges, and management options," Journal Carcinogenesis, vol. 14, no. 1, p. 3, 2015.

[8] R. Moran and M. Lennon, "Endoscopic ultrasonography in the evaluation of pancreatic cysts (p 185-192)," in 
Endosonography, R. Hawes, P. Fockens, and S. Varadajulu, Eds., Elsevier, Philadelphia, 4th Ed. edition, 2018.

[9] M. Distler, D. Aust, J. Weitz, C. Pilarsky, and R. Grützmann, "Precursor lesions for sporadic pancreatic cancer: PanIN, IPMN, and MCN," BioMed Research International, vol. 2014, Article ID 474905, 11 pages, 2014.

[10] European Study Group on Cystic Tumours of the Pancreas, "European evidence-based guidelines on pancreatic cystic neoplasms," Gut, vol. 67, no. 5, pp. 789-804, 2018.

[11] R. Repák, S. Rejchrt, J. Bártová, E. Malírová, V. Tycová, and J. Bures, "Endoscopic ultrasonography (EUS) and EUS-guided fine-needle aspiration with cyst fluid analysis in pancreatic cystic neoplasms," Hepato-Gastroenterology, vol. 56, no. 91-92, pp. 629-635, 2009.

[12] J. M. Dumonceau, M. Polkowski, A. Larghi et al., "Indications, results, and clinical impact of endoscopic ultrasound (EUS)guided sampling in gastroenterology: European Society of Gastrointestinal Endoscopy (ESGE) Clinical Guideline," Endoscopy, vol. 43, no. 10, pp. 897-912, 2011.

[13] M. Polkowski, C. Jenssen, P. Kaye et al., “Technical aspects of endoscopic ultrasound (EUS)-guided sampling in gastroenterology: European Society of Gastrointestinal Endoscopy (ESGE) Technical Guideline - March 2017," Endoscopy, vol. 49, no. 10, pp. 989-1006, 2017.

[14] A. Gillis, I. Cipollone, G. Cousins, and K. Conlon, "Does EUS-FNA molecular analysis carry additional value when compared to cytology in the diagnosis of pancreatic cystic neoplasm? A systematic review," HPB: The Official Journal of the International Hepato Pancreato Biliary Association, vol. 17, no. 5, pp. 377-386, 2015.

[15] W. R. Brugge, K. Lewandrowski, E. Lee-Lewandrowski et al., "Diagnosis of pancreatic cystic neoplasms: a report of the cooperative pancreatic cyst study," Gastroenterology, vol. 126, no. 5, pp. 1330-1336, 2004.

[16] C. Guarner-Argente, P. Shah, A. Buchner, N. A. Ahmad, M. L. Kochman, and G. G. Ginsberg, "Use of antimicrobials for EUS-guided FNA of pancreatic cysts: a retrospective, comparative analysis," Gastrointestinal Endoscopy, vol. 74, no. 1, pp. 81-86, 2011.

[17] A. Facciorusso, V. R. Buccino, A. Turco, M. Antonino, and N. Muscatiello, "Antibiotics do not decrease the rate of infection after endoscopic ultrasound fine-needle aspiration of pancreatic cysts," Digestive Diseases and Sciences, vol. 64, no. 8, pp. 2308-2315, 2019.

[18] V. M. Castellano-Megías, C. I. Andrés, G. López-Alonso, and F. Colina-Ruizdelgado, "Pathological features and diagnosis of intraductal papillary mucinous neoplasm of the pancreas," World Journal Gastrointestinal Oncology, vol. 6, no. 9, pp. 311-324, 2014.

[19] R. Grützmann, M. Niedergethmann, C. Pilarsky, G. Klöppel, and H. D. Saeger, "Intraductal papillary mucinous tumors of the pancreas: biology, diagnosis, and treatment," The Oncologist, vol. 15, no. 12, pp. 1294-1309, 2010.

[20] T. Furukawa, G. Klöppel, N. Volkan Adsay et al., "Classification of types of intraductal papillary-mucinous neoplasm of the pancreas: a consensus study," Virchows Archiv, vol. 447, no. 5, pp. 794-799, 2005.

[21] T. Furukawa, T. Hatori, I. Fujita et al., "Prognostic relevance of morphological types of intraductal papillary mucinous neoplasms of the pancreas," Gut, vol. 60, no. 4, pp. 509-516, 2011.
[22] M. Tanaka, C. Fernández-del Castillo, V. Adsay et al., "International consensus guidelines 2012 for the management of IPMN and MCN of the pancreas," Pancreatology, vol. 12, no. 3, pp. 183-197, 2012.

[23] N. V. Adsay, K. Merati, O. Basturk et al., "Pathologically and biologically distinct types of epithelium in intraductal papillary mucinous neoplasms: delineation of an "intestinal" pathway of carcinogenesis in the pancreas," The American Journal of Surgical Pathology, vol. 28, no. 7, pp. 839-848, 2004.

[24] N. V. Adsay, C. Pierson, F. Sarkar et al., "Colloid (mucinous noncystic) carcinoma of the pancreas," The American Journal of Surgical Pathology, vol. 25, no. 1, pp. 26-42, 2001.

[25] P. J. Allen, "The diagnosis and management of cystic lesions of the pancreas," Chinese Clinical Oncology, vol. 6, no. 6, p. 60, 2017.

[26] D. V. Sahani, R. Kadavigere, A. Saokar, C. Fernandez-del Castillo, W. R. Brugge, and P. F. Hahn, "Cystic pancreatic lesions: a simple imaging-based classification system for guiding management," Radiographics, vol. 25, no. 6, pp. 1471-1484, 2005.

[27] S. Springer, Y. Wang, M. Dal Molin et al., "A combination of molecular markers and clinical features improve the classification of pancreatic cysts," Gastroenterology, vol. 149, no. 6, pp. 1501-1510, 2015.

[28] P. S. Moore, G. Zamboni, A. Brighenti et al., "Molecular characterization of pancreatic serous microcystic adenomas: evidence for a tumor suppressor gene on chromosome 10q," The American Journal of Pathology, vol. 158, no. 1, pp. 317-321, 2001.

[29] B. Jais, V. Rebours, G. Malleo et al., "Serous cystic neoplasm of the pancreas: a multinational study of 2622 patients under the auspices of the International Association of Pancreatology and European Pancreatic Club (European Study Group on Cystic Tumors of the Pancreas)," Gut, vol. 65, no. 2, pp. 305-312, 2016.

[30] L. A. van der Waaij, H. M. van Dullemen, and R. J. Porte, "Cyst fluid analysis in the differential diagnosis of pancreatic cystic lesions: a pooled analysis," Gastrointestinal Endoscopy, vol. 62, no. 3, pp. 383-389, 2005.

[31] D. S. Klimstra, B. M. Wenig, and C. S. Heffess, "Solid-pseudopapillary tumor of the pancreas: a typically cystic carcinoma of low malignant potential," Seminars in Diagnostic Pathology, vol. 17, no. 1, pp. 66-80, 2000.

[32] M. Tanaka, S. Chari, V. Adsay et al., "International consensus guidelines for management of intraductal papillary mucinous neoplasms and mucinous cystic neoplasms of the pancreas," Pancreatology, vol. 6, no. 1-2, pp. 17-32, 2006.

[33] M. Tanaka, C. Fernández-Del Castillo, T. Kamisawa et al., "Revisions of international consensus Fukuoka guidelines for the management of IPMN of the pancreas," Pancreatology, vol. 17, no. 5, pp. 738-753, 2017.

[34] S. S. Vege, B. Ziring, R. Jain, and P. Moayyedi, "American gastroenterological association institute guideline on the diagnosis and management of asymptomatic neoplastic pancreatic cysts," Gastroenterology, vol. 148, no. 4, pp. 819-822, 2015, quize12-3.

[35] J. R. Aparicio, J. Martínez, M. Niveiro et al., "Direct intracystic biopsy and pancreatic cystoscopy through a 19-gauge needle EUS (with videos)," Gastrointestinal Endoscopy, vol. 72, no. 6, pp. 1285-1288, 2010.

[36] L. Barresi, I. Tarantino, D. Ligresti, G. Curcio, A. Granata, and M. Traina, "A new tissue acquisition technique in pancreatic 
cystic neoplasm: endoscopic ultrasound-guided through-theneedle forceps biopsy," Endoscopy, vol. 47, no. S 01, pp. E297-E298, 2015.

[37] K. D. Pham, T. Engjom, H. Gjelberg Kollesete, and L. Helgeland, "Diagnosis of a mucinous pancreatic cyst and resection of an intracystic nodule using a novel through-theneedle micro forceps," Endoscopy, vol. 48, no. S 01, pp. E125-E126, 2016.

[38] M. H. Shakhatreh, S. R. Naini, A. A. Brijbassie, D. J. Grider, P. Shen, and P. Yeaton, "Use of a novel through-the-needle biopsy forceps in endoscopic ultrasound," Endoscopy International Open, vol. 04, no. 04, pp. E439-E442, 2016.

[39] F. Attili, D. Pagliari, M. Rimbaş et al., "Endoscopic ultrasound-guided histological diagnosis of a mucinous non-neoplastic pancreatic cyst using a specially designed through-the-needle microforceps," Endoscopy, vol. 48, no. S 01, pp. E188-E189, 2016.

[40] A. Huelsen, C. Cooper, N. Saad, and S. Gupta, "Endoscopic ultrasound-guided, through-the-needle forceps biopsy in the assessment of an incidental large pancreatic cystic lesion with prior inconclusive fine-needle aspiration," Endoscopy, vol. 49, no. S 01, pp. E109-E110, 2017.

[41] A. L. Chen, J. Misdraji, W. R. Brugge, C. R. Ferrone, and M. B. Pitman, "Acinar cell cystadenoma: a challenging cytology diagnosis, facilitated by moray ${ }^{\circledR}$ micro-forceps biopsy," Diagnostic Cytopathology, vol. 45, no. 6, pp. 557-560, 2017.

[42] O. Basar and W. R. Brugge, "My treatment approach: pancreatic cysts," Mayo Clinic Proceedings, vol. 92, no. 10, pp. 15191531, 2017.

[43] F. Vilas-Boas and G. Macedo, "Pancreatic cystic lesions: new endoscopic trends in diagnosis," Journal of Clinical Gastroenterology, vol. 52, no. 1, pp. 13-19, 2018.

[44] O. Basar, "Pancreatic cyst biopsy: improvement in diagnosis with micro forceps biopsy," Cancer Cytopathology, vol. 126, no. 4 , pp. 227-228, 2018.

[45] C. Mittal, J. C. Obuch, H. Hammad et al., "Technical feasibility, diagnostic yield, and safety of microforceps biopsies during EUS evaluation of pancreatic cystic lesions (with video)," Gastrointestinal Endoscopy, vol. 87, no. 5, pp. 1263-1269, 2018.

[46] O. Basar, O. Yuksel, D. J. Yang et al., "Feasibility and safety of microforceps biopsy in the diagnosis of pancreatic cysts," Gastrointestinal Endoscopy, vol. 88, no. 1, pp. 79-86, 2018.

[47] M. L. Zhang, R. N. Arpin, W. R. Brugge, D. G. Forcione, O. Basar, and M. B. Pitman, "Moray micro forceps biopsy improves the diagnosis of specific pancreatic cysts," Cancer Cytopathology, vol. 126, no. 6, pp. 414-420, 2018.

[48] B. Kovacevic, J. G. Karstensen, R. F. Havre et al., "Initial experience with EUS-guided microbiopsy forceps in diagnosing pancreatic cystic lesions: a multicenter feasibility study (with video)," Endoscopic Ultrasound, vol. 7, no. 6, pp. 383-388, 2018.

[49] M. Minsky, "Memoir on inventing the confocal scanning microscope," Scanning, vol. 10, no. 4, 138 pages, 1988.

[50] T. C. Savidge, J. A. Walker-Smith, and A. D. Phillips, "Novel insights into human intestinal epithelial cell proliferation in health and disease using confocal microscopy," Gut, vol. 36, no. 3, pp. 369-374, 1995.

[51] ASGE Technology Committee, "Confocal laser endomicroscopy," Gastrointestinal Endoscopy, vol. 80, no. 6, pp. 928938, 2014.
[52] A. Hoffman, M. Goetz, M. Vieth, P. R. Galle, M. F. Neurath, and R. Kiesslich, "Confocal laser endomicroscopy: technical status and current indications," Endoscopy, vol. 38, no. 12, pp. 1275-1283, 2006.

[53] M. Kunes, J. Kvetina, J. Malakova, J. Bures, M. Kopacova, and S. Rejchrt, "Pharmacokinetics and organ distribution of fluorescein in experimental pigs: an input study for confocal laser endomicroscopy of the gastrointestinal tract," Neuro Endocrinology Letters, vol. 31, Suppl 2, pp. 57-61, 2010.

[54] A. Tringali, A. Lemmers, V. Meves et al., "Intraductal biliopancreatic imaging: European Society of Gastrointestinal Endoscopy (ESGE) technology review," Endoscopy, vol. 47, no. 08, pp. 739-753, 2015.

[55] F. Li, S. El-Dika, R. M. Modi, W. Chen, and S. G. Krishna, "Poorly differentiated pancreatic carcinoma with sarcomatoid differentiation: confocal endomicroscopy of an uncommon pancreatic cystic lesion," Endoscopy, vol. 48, no. S 01, pp. E363-E364, 2016.

[56] B. Napoleon, M. Palazzo, A. I. Lemaistre et al., "Needle-based confocal laser endomicroscopy of pancreatic cystic lesions: a prospective multicenter validation study in patients with definite diagnosis," Endoscopy, 2018.

[57] F. Salom and F. Prat, "Current indications and yield of endoscopic ultrasound and ancillary techniques in pancreatic cystic neoplasms," Clinical Journal of Gastroenterology, vol. 12, no. 2, pp. 93-101, 2019.

[58] N. Okuno, K. Hara, and M. Obata, "Novel method of diagnosing solid pseudopapillary neoplasms of the pancreas: needle-based confocal laser endomicroscopy," Digestive Endoscopy, 2019.

[59] J. E. East, J. L. Vleugels, P. Roelandt et al., "Advanced endoscopic imaging: European Society of Gastrointestinal Endoscopy (ESGE) Technology Review," Endoscopy, vol. 48, no. 11, pp. 1029-1045, 2016.

[60] A. Fugazza, F. Gaiani, M. C. Carra et al., "Confocal laser endomicroscopy in gastrointestinal and pancreatobiliary diseases: a systematic review and meta-analysis," Biomed Research International, vol. 2016, Article ID 4638683, 31 pages, 2016.

[61] V. J. A. Konda, H. R. Aslanian, M. B. Wallace, U. D. Siddiqui, J. Hart, and I. Waxman, "First assessment of needle-based confocal laser endomicroscopy during EUS-FNA procedures of the pancreas (with videos)," Gastrointestinal Endoscopy, vol. 74, no. 5, pp. 1049-1060, 2011.

[62] V. J. Konda, A. Meining, L. H. Jamil et al., "A pilot study of in vivo identification of pancreatic cystic neoplasms with needle-based confocal laser endomicroscopy under endosonographic guidance," Endoscopy, vol. 45, no. 12, pp. 1006-1013, 2013.

[63] M. Kahaleh, B. G. Turner, K. Bezak et al., "Probe-based confocal laser endomicroscopy in the pancreatic duct provides direct visualization of ductal structures and aids in clinical management," Digestive and Liver Disease, vol. 47, no. 3, pp. 202-204, 2015.

[64] Y. Nakai, T. Iwashita, D. H. Park, J. B. Samarasena, J. G. Lee, and K. J. Chang, "Diagnosis of pancreatic cysts: EUS-guided, through-the-needle confocal laser-induced endomicroscopy and cystoscopy trial: DETECT study," Gastrointestinal Endoscopy, vol. 81, no. 5, pp. 1204-1214, 2015.

[65] B. Napoleon, A. I. Lemaistre, B. Pujol et al., "A novel approach to the diagnosis of pancreatic serous cystadenoma: needle- 
based confocal laser endomicroscopy," Endoscopy, vol. 47, no. 01 , pp. 26-32, 2015.

[66] A. Kadayifci, M. Atar, O. Basar, D. G. Forcione, and W. R. Brugge, "Needle-based confocal laser endomicroscopy for evaluation of cystic neoplasms of the pancreas," Digestive Diseases and Sciences, vol. 62, no. 5, pp. 1346-1353, 2017.

[67] A. Kadayifci, M. Atar, M. Yang, C. Fernandez-Del Castillo, M. Mino-Kenudson, and W. R. Brugge, "Imaging of pancreatic cystic lesions with confocal laser endomicroscopy: an ex vivo pilot study," Surgical Endoscopy, vol. 31, no. 12, pp. 51195126, 2017.

[68] S. G. Krishna, R. M. Modi, A. K. Kamboj et al., "In vivo and ex vivo confocal endomicroscopy of pancreatic cystic lesions: a prospective study," World Journal of Gastroenterology, vol. 23, no. 18, pp. 3338-3348, 2017.

[69] K. Karia, I. Waxman, V. J. Konda et al., "Needle-based confocal endomicroscopy for pancreatic cysts: the current agreement in interpretation," Gastrointestinal Endoscopy, vol. 83, no. 5, pp. 924-927, 2016.

[70] S. G. Krishna, W. R. Brugge, J. M. Dewitt et al., "Needle-based confocal laser endomicroscopy for the diagnosis of pancreatic cystic lesions: an international external interobserver and intraobserver study (with videos)," Gastrointestinal Endoscopy, vol. 86, no. 4, pp. 644-654.e2, 2017.

[71] M. B. Wallace, A. Meining, M. I. Canto et al., "The safety of intravenous fluorescein for confocal laser endomicroscopy in the gastrointestinal tract," Alimentary Pharmacology \& Therapeutics, vol. 31, no. 5, pp. 548-552, 2010.

[72] C. Le Pen, L. Palazzo, and B. Napoleon, “A health economic evaluation of needle-based confocal laser endomicroscopy for the diagnosis of pancreatic cysts," Endoscopy International Open, vol. 05, no. 10, pp. E987-E995, 2017. 


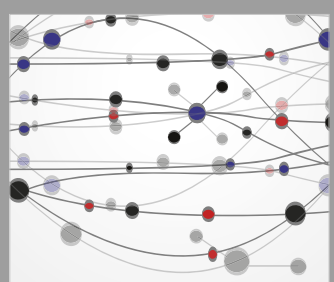

The Scientific World Journal
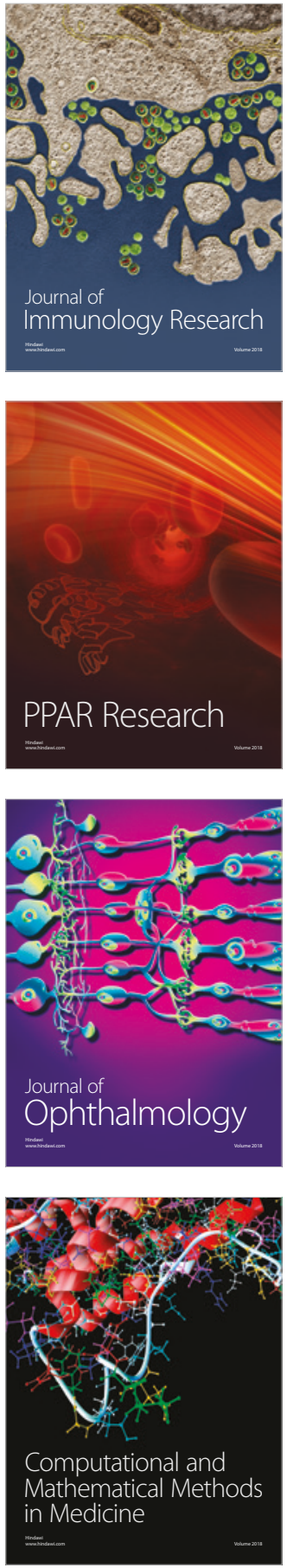

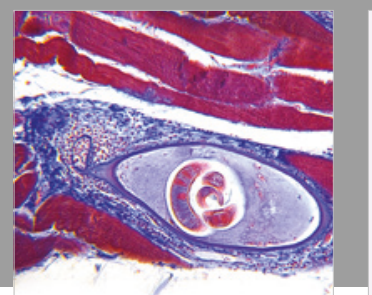

Gastroenterology Research and Practice

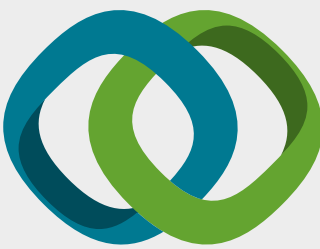

\section{Hindawi}

Submit your manuscripts at

www.hindawi.com
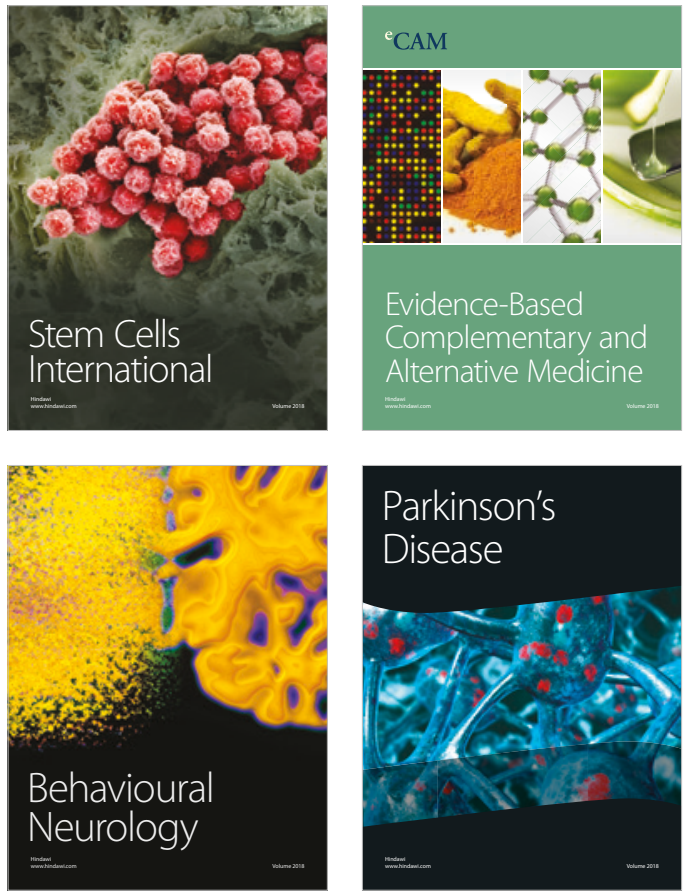

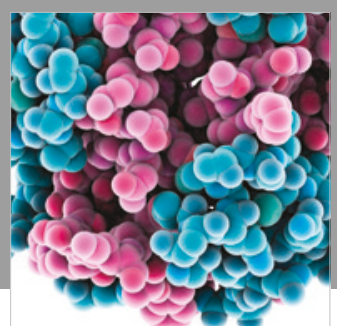

ournal of

Diabetes Research

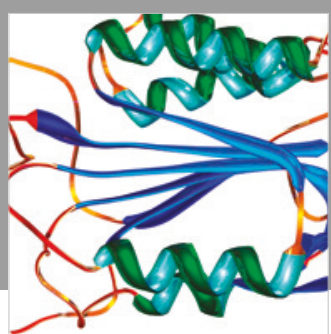

Disease Markers
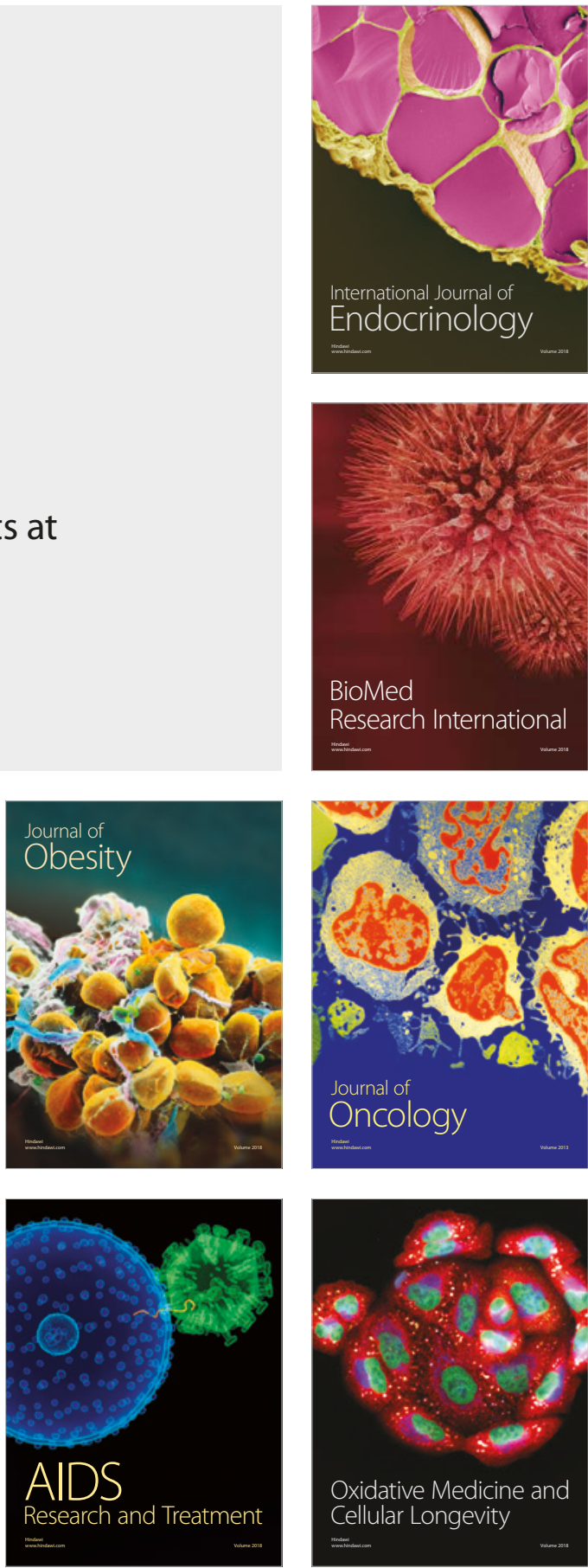